\title{
Ganadería en Colombia, generando alternativas sostenibles
}

\section{Livestock in Colombia, generating sustainable alternatives}

\author{
Rafael Zavala Gómez del Campo ${ }^{1}$ \\ ${ }^{1}$ Representante FAO en Colombia \\ (México) Licenciado en Zootecnia de la Universidad Autónoma Metropolitana, con \\ Maestría en Agricultura Sostenible en el Wye Collage de la Universidad de \\ Londres, y un Doctorado en Políticas para el Desarrollo Rural en la Universidad de \\ East Anglia, Inglaterra \\ Ponencia II Congreso Internacional de Ciencias Veterinarias y Zootécnicas \\ rafael.zavala@fao.org
}

Recibido 12 de Febrero 2015, Aceptado 10 de Abril 2015

\section{RESUMEN}

Se realiza una síntesis sobre los principales obstáculos para intensificar eficientemente la ganadería en Colombia y América Latina. Se analiza el desarrollo del sector pecuario en sus circunstancias actuales y las expectativas futuras que han generado la revaloración de los recursos naturales teniendo en cuenta el incremento las exportaciones de sus productos en los últimos 10 años, lo cual podría convertirse en factor del desarrollo económico. En respuesta al reto ambiental que genera el incremento de la producción ganadera, especialmente en lo que se refiere a tasas de deforestación, se están presentando algunos cambios hacia sistemas combinados más intensivos de cultivos y ganadería con mayor producción de lácteos. Todo esto se debe a: inversiones en infraestructura de transporte, conversión de tierras de pastoreo en tierras de labranza y el rápido aumento del consumo per cápita de carne y leche ha sido acompañado de un cambio en las fuentes de calorías y proteínas, reemplazando las carnes rojas por las de aves de corral. La tendencia hacia sistemas de producción pecuarios más intensivos, está convirtiendo a la región en una de las más sensibles del mundo en términos de riesgos sanitarios para los animales, por lo tanto podría concluirse que la discrepancia entre el desarrollo de la ganadería y su impacto ambiental requiere un enfoque decidido pero equilibrado, considerando inversiones en 
investigaciones, prevención y erradicación de enfermedades, educación y capacitación y una serie de medidas para mejorar la productividad y la rentabilidad de la actividad.

Palabras clave: Políticas, ganadería sostenible, silvopastoriles, seguridad alimentaria.

\section{ABSTRACT}

A summary of the main obstacles to efficiently enhance livestock in Colombia and Latin America is performed. The development of livestock in their current circumstances and future expectations that have generated the appreciation of natural resources taking into account the increase in exports of their products in the past 10 years, which could become a factor of economic development is analyzed. In response to environmental challenge that generates increased livestock production, particularly as regards deforestation rates are showing some changes toward more intensive systems combined crop and livestock production more milk. All this is due to: investments in transportation infrastructure, land conversion grazing on farmland and the rapid increase in per capita consumption of meat and milk has been accompanied by a shift in the sources of calories and protein, replacing meat red by poultry. The trend toward more intensive livestock production is making the region one of the most sensitive in the world in terms of health risks to animals could therefore be concluded that the discrepancy between livestock development and its environmental impact requires a strong but balanced approach, considering investments in research, prevention and eradication, education and training and a series of measures to improve productivity and profitability of the activity.

Keywords: Policy, sustainable farming, forest grazing, food security.

\section{RESUMO}

Um resumo dos principais obstáculos para melhorar a eficiência de gado na Colômbia e na América Latina é realizada. O desenvolvimento da pecuária em 
suas circunstâncias atuais e expectativas futuras que têm gerado a valorização dos recursos naturais, tendo em conta o aumento das exportações de seus produtos nos últimos 10 anos, o que poderia se tornar um fator de desenvolvimento econômico é analisada. Em resposta ao desafio ambiental que gera o aumento da produção de gado, nomeadamente no que respeita às taxas de desmatamento estão mostrando algumas mudanças em direção a sistemas mais intensivos produção agrícola e pecuária mais leite combinadas. Tudo isso é devido a: investimentos em infra-estrutura de transporte, a conversão de pastagens em terras agrícolas e do rápido aumento do consumo per capita de carne e leite tem sido acompanhado por uma mudança nas fontes de calorias e proteínas, substituindo a carne Red de aves. A tendência de maior produção pecuária intensiva está tornando a região um dos mais sensíveis no mundo em termos de riscos para a saúde dos animais poderiam, portanto, concluir que a discrepância entre o desenvolvimento da pecuária e do seu impacto ambiental requer uma abordagem forte, mas equilibrada, considerando os investimentos em pesquisa, prevenção e erradicação, educação e formação e de uma série de medidas para melhorar a produtividade e lucratividade da atividade.

Palavras-chave: Política, agricultura sustentável, pastagem florestal, segurança alimentar.

\section{INTRODUCCIÓN}

La disponibilidad de alimentos ha sido siempre una preocupación fundamental del ser humano. En América Latina los pequeños productores agropecuarios son los responsables de la mayor parte de los alimentos que se consumen en los países en desarrollo, tal es el caso de Colombia, en donde se cuenta con una población bovina de 22.6 millones de bovinos, siendo los terneros el $17.82 \%$, vacas (mayores 1 año) 56\%, machos (mayores a 1 año) 26,16\%, el número de predios ganaderos supera los 500.000 , y cuya población tiene un consumo per cápita de $20.8 \mathrm{~kg}$ de carne bovina (FEDEGAN, 2013). 
De otra parte, las circunstancias presentes y las expectativas futuras acerca del curso de la producción agropecuaria han generado la revaloración de los recursos naturales con potencial agrícola. Esto es de especial significancia para un país que, como Colombia, tiene, junto a un grupo no muy amplio de naciones, una dotación de recursos agrícolas no explotados plenamente, más aún, las perspectivas de crecimiento de la demanda mundial de alimentos para el año 2050, se estima que, en las naciones exportadoras, la agricultura podría convertirse en un pilar central del desarrollo económico. De esta forma, en el caso colombiano, la dinamización de los productos no tradicionales con potencial exportador, en razón a su capacidad competitiva que pueden exhibir en los mercados mundiales, una decisión eficaz en términos productivos y de integración comercial, dada la alta utilización de mano de obra y la presencia importante de pequeños productores, representaría una medida de política que enfrentaría de manera efectiva los problemas de subempleo, informalidad y pobreza que afectan al sector rural colombiano y que, hasta el presente, el enfoque de desarrollo y las políticas públicas no han podido resolver (FEDESARROLLO et al., 2013).

\section{TENDENCIAS DE LA GANADERÍA EN AMÉRICA LATINA}

La producción de carne y leche ha incrementado en el orden de $22 \%$ en los últimos 10 años en Latinoamérica, superando las tasas de crecimiento de Estados Unidos y Europa. Los resultados actuales indican un mayor porcentaje de producción mundial de carne de vacuno, cordero y ave en comparación con Estados Unidos y casi la misma proporción en términos de la producción mundial de leche (FAO, 2013).

Los consumidores están prefiriendo diversas fuentes de proteína animal, entre ellas pollo, cerdo, huevos y productos lácteos, sobre el vacuno y el cordero. El crecimiento de las industrias avícola y porcina y por tanto su consumo han sido fenómenos relevantes en el desarrollo de la industria pecuaria de América Latina. El consumo per cápita de pollo aumentó en varios países de la región, entre ellos Brasil, Argentina, Chile y México, donde la oferta de fuentes alternativas de 
proteínas disponibles para el consumo se ha reducido sobre una base per cápita (Figura 1) (CEPAL et al., 2012)

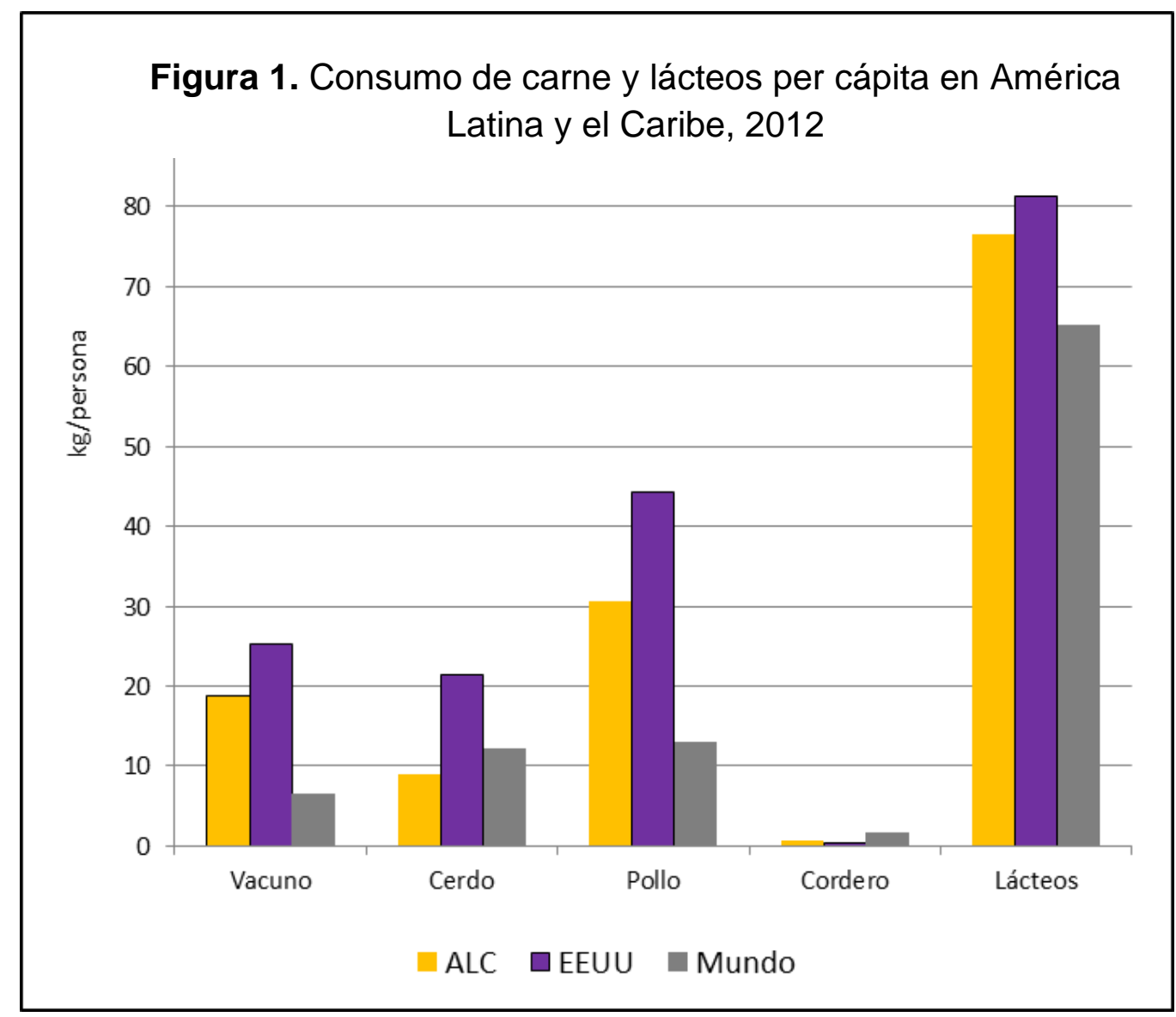

Las nuevas tecnologías y prácticas de producción, incluidas razas mejoradas, han sido adoptadas ampliamente durante la última década, lo cual ha generado un sólido aumento en la producción de carne y leche por cabeza en todas las especies de ganado. La productividad de carne de cerdo y ave está alcanzando niveles parecidos a los de los EE.UU. y se ubica considerablemente sobre el promedio mundial en ambos mercados. Sin embargo, la productividad láctea (1.55 toneladas/cabeza/año) continúa considerablemente a la zaga respecto de EE.UU. (9.31 toneladas/cabeza/año), pero está sobre el promedio mundial (1.10 toneladas/cabeza/año). No obstante, se ha ido cerrando paulatinamente la brecha; Latinoamérica logró aumentar su producción más de $22 \%$ durante la última década en comparación con EE.UU. que alcanzó un crecimiento de alrededor del $16 \%(F A O, 2013)$. 
Respondiendo al reto ambiental que genera el incremento de la producción ganadera, especialmente en lo que se refiere a tasas de deforestación, se están presentando algunos cambios en los países latinoamericanos hacia sistemas combinados más intensivos de cultivos y ganadería con mayor producción de lácteos, gracias a inversiones en infraestructura de transporte y la conversión de tierras de pastoreo en tierras de labranza (Wassenaar et al., 2007). Además, el rápido aumento del consumo per cápita de carne y leche ha sido acompañado de un cambio dietario en las fuentes de calorías y proteínas que ha llevado a que las carnes rojas sean reemplazadas por carne de aves de corral (FAO, 2013).

\section{PERSPECTIVAS DE LA GANADERÍA EN AMÉRICA LATINA}

Se tiene previsto que durante la próxima década la producción de carne continuará su rápido crecimiento, aunque a una tasa anual menor, donde los factores clave en el rendimiento esperado incluyen ventajas comparativas de la ganadería extensiva en Suramérica, previendo un crecimiento en los ingresos y la demanda de carne de ave superior a la del vacuno, con el fin de fomentar políticas de producción y mitigación del impacto ambiental (OCDE y FAO, 2014).

Se prevé que la ventaja comparativa de América Latina es la producción de ganado a partir de su potencial de extensión de los pastizales que continuará ejerciendo presión para despejar bosques. Además, el crecimiento rápido en la producción de aves, cerdos y leche, posiblemente creará más presiones para despejar zonas forestales y dar paso a cultivos forrajeros, lo que bajo los actuales esquemas de producción la perdida de bosques es proporcional al crecimiento de la áreas de cultivo de forraje (OCDE y FAO, 2011).

Se espera que la producción de leche crezca en $21.2 \%$ en Latinoamérica durante los próximos 10 años (Figura 2), en comparación al incremento de $25 \%$ durante la década anterior. Los altos precios de la energía y del forraje continuarán favoreciendo la ventaja comparativa de producción de leche en pastizales que impera en América Latina por sobre los sistemas basados en alimento con granos 
de los países desarrollados, lo que reducirá aún más las importaciones netas de lácteos de la región (OCDE y FAO, 2011).

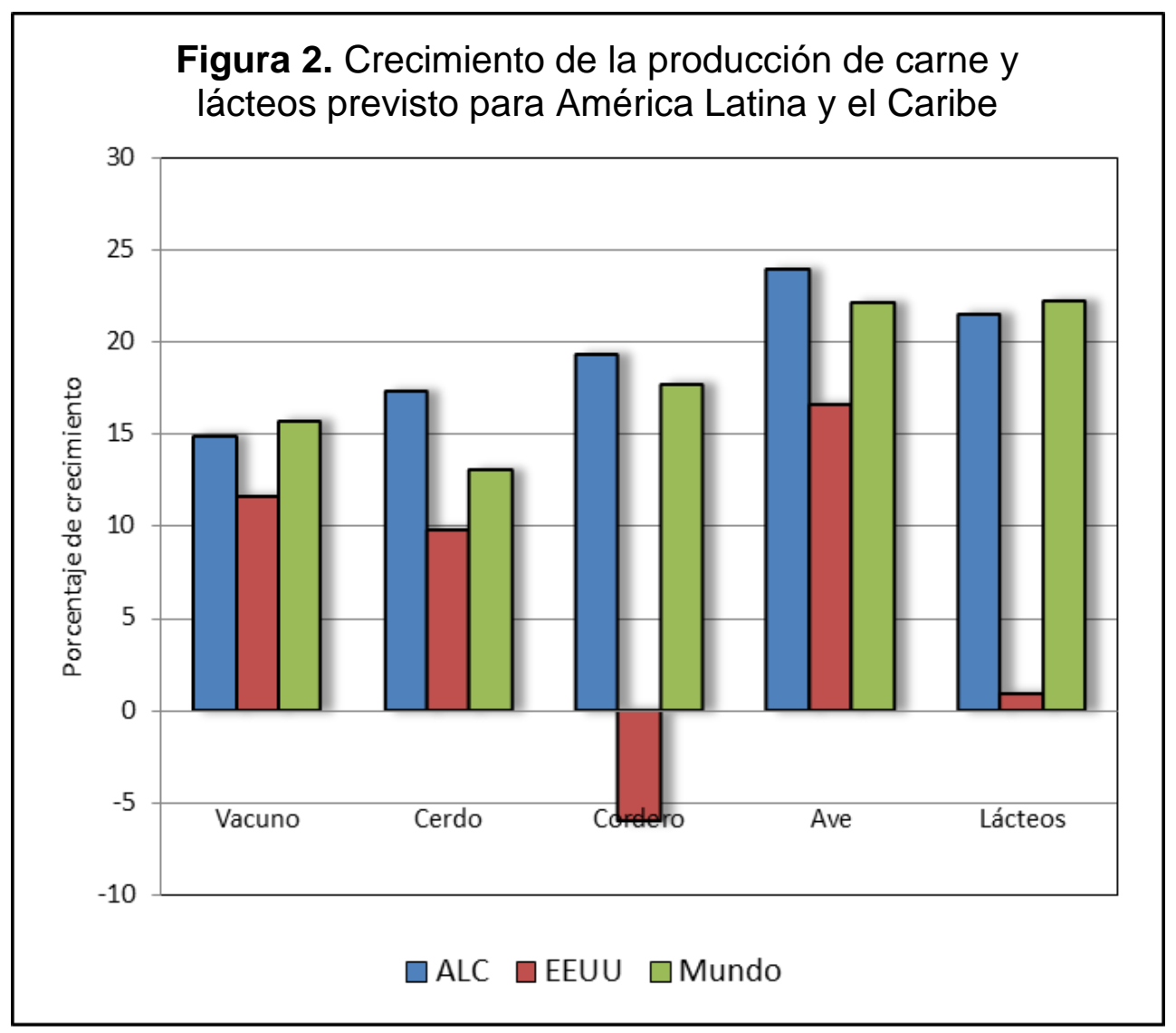

Fuente: OECD y FAO, (2012)

Los agricultores de bajos recursos económicos por lo general crían animales de corral como cerdos, aves, ovejas y cabras en lugar de ganado vacuno por diversos motivos, incluidas la menor inversión de capital requerida y la mayor eficiencia en términos de producción de carne (Otte et al., 2005). En este sentido, es previsible que el rápido crecimiento de la producción ganadera y de carne mejore las condiciones de vida de muchos pequeños productores rurales de la región en el futuro.

La tendencia hacia sistemas de producción pecuarios más intensivos en América Latina está convirtiendo a la región en una de las más sensibles del mundo en términos de riesgos sanitarios para los animales, de manera similar su aumento e 
intensificación creará una demanda creciente de productos farmacéuticos y servicios veterinarios (Perry et al., 2011).

\section{RECOMENDACIONES DE POLÍTICAS PARA LA GANADERÍA EN COLOMBIA}

Para poder fomentar el crecimiento del sector ganadero reduciendo los costos ambientales, es indispensable flexibilizar las restricciones que impiden su desarrollo, puesto que este sector tiene el potencial de reducir la pobreza en la región, lo cual requiere incentivos adecuados y una cuidadosa planificación para evitar que los pequeños productores queden rezagados, además programas de integración, a la cadena de suministro ganadero (Pica et al., 2008).

Las políticas para el sector ganadero deben estar encaminadas a mejorar el acceso a insumos de producción (tierra, agua, herramientas de gestión de riesgos, entre otros); fomentar la eficiencia y producción de los pequeños agricultores incluyendo medidas para mejorar los accesos a créditos, servicios veterinarios para erradicar enfermedades que pueden generar dificultades económicas, prestar servicios de extensión para ofrecer la capacitación y asistencia técnica, en reproducción, marketing, administración y adopción de nuevas tecnologías; para generar mejor calidad de productos, estimulando la competitividad a través de investigaciones y asistencia con fondos públicos, con el fin, que los pequeños productores puedan cumplir normas nacionales, regionales e internacionales en materia de calidad. También es importante implementar: programas de conservación de suelos, silvopastoreo y de pastoreo rotacional, así como de eficiencia sostenible en cultivos y ganadería para reducir la deforestación y degradación (pastizales), promoviendo sistemas intensivos para mejorar el ciclaje de nutrientes y calidad del agua; y finalmente políticas para la protección de aéreas silvestres incorporando sistemas de producción pecuaria a los esfuerzos de mantener los paisaje naturales para disminuir la pérdida de biodiversidad (IICA, 2013).

Además de las políticas mencionadas anteriormente, se deben generar mecanismos eficaces de fijación de precios que fomenten conductas adecuadas 
en términos medioambientales que sean económicamente factibles, y medidas para reducir los brotes de enfermedades animales al mínimo, tales como sistemas integrales de trazabilidad, esto con el fin de aprovechar oportunidades del crecimiento de la demanda mundial por alimentos. También se debe promover políticas para investigación y adaptación de la agricultura al cambio climático, para una mayor utilización de la biomasa como fuente de energía, reforzando estrategias para asegurar inclusión de agricultura familiar (Steinfeld et al., 2006).

\section{PROGRAMA MUNDIAL DE GANADERÍA SOSTENIBLE}

La ganadería en el desarrollo sostenible tiene tres ejes: 1) equidad y desarrollo económico, 2) recursos naturales y cambio climático, y 3) seguridad alimentaria y salud.

\section{Equidad y desarrollo económico}

El $60 \%$ de las viviendas rurales en los países en vías del desarrollo mantienen ganado, 1.7 mil millones de habitantes dependen de la ganadería, hay 530 millones personas "tenedores" de ganado, que se califican en pobreza extrema, tristemente el pequeño productor está siendo desplazado, además este grupo es particularmente vulnerable al impacto del cambio climático. Por otro lado el $70 \%$ de la demanda global de carne bovina, se debe al crecimiento poblacional y la urbanización debido al incremento en el poder adquisitivo (Thornton et al., 2002). La mayor eficiencia en el desarrollo de la avicultura y porcicultura en comparación con los rumiantes es bastante desigual, por ejemplo, la producción de pollo en Latinoamérica creció a una tasa sorprendente de $84.8 \%$ entre 2000 y 2011 y hoy en día da cuenta del 23\% del total mundial (CEPAL et al., 2013),

La rápida adopción y distribución de tecnologías más eficientes de producción en aves y cerdos ha permitido a muchos países aumentar las exportaciones de fuentes tradicionales de proteínas, como el vacuno, y reemplazar la creciente oferta de aves y cerdo en el consumo nacional. Por otro lado, los países de América Latina han sido principalmente consumidores netos de productos lácteos y constituyen casi el 15\% de las importaciones mundiales de leche en polvo entera 
y descremada (OCDE y FAO, 2012). El rápido crecimiento demográfico de la región ha intensificado la demanda de estos productos, pero las crisis económicas que afectan a muchas naciones han dado lugar a patrones de importación erráticos (Blayney et al., 2006). Es de anotar que de todas las tierras potencialmente aptas para expandir la producción ganadera y agrícola, alrededor del $28 \%$ se encuentra en América Latina, más que en ninguna otra región, a excepción de África (Nash, 2012).

\section{Seguridad alimentaria y salud}

En América Latina los productos de la ganadería representan el $26 \%$ de la proteína en la dieta global, correspondiente al $13 \%$ de las calorías, mientras que en países desarrollados aportan más del 40\%, además la ganadería tiene funciones múltiples tales como aportaciones adicionales al cultivo y hortalizas, representados como estiércol y/o abono, generando un valor adicional en cueros, lana y harinas, también aporta los nutrientes esenciales a los grupos más vulnerables de la sociedad como lo son niños y maternas.

Los brotes de enfermedades animales frecuentes $y$ en aumento afectan negativamente el desarrollo de los sistemas de producción de ganado en América Latina y a menudo plantean graves riesgos para la salud humana. La región ocupa un lugar destacado en la lista de lugares donde la fiebre aftosa complica los esfuerzos por establecer industrias ganaderas rentables y sostenibles. Un reciente brote de esta enfermedad en Paraguay habría provocado decenas de millones en pérdidas, debido al sacrificio del ganado y la imposibilidad de cursar las exportaciones de vacuno (UPI, 2012). El contagio de fiebre aftosa también implica dificultades económicas, particularmente para los pequeños productores latinoamericanos, que pierden los pocos animales que crían como principal fuente de ingresos y proteínas. En este sentido, la FAO y la Organización Mundial de Sanidad Animal (OIE) anunciaron recientemente que aunaron fuerzas para combatir la fiebre aftosa a nivel mundial (FAO, 2013). 
Algunas de las enfermedades más frecuentes y persistentes de la industria del cerdo en América Latina son el Circovirus Porcino (PCV2), el Teschovirus, el Paramixovirus, la fiebre porcina clásica (CSF) y la gastroenteritis. La CSF ha resultado ser muy persistente, con brotes recientes en Brasil, Guatemala y Nicaragua, pese al Programa Intercontinental de la FAO diseñado para erradicar el mal en 2020 (Martins, 2011). La influenza aviar no se ha convertido en una epidemia en la región, pero la preocupación sigue vigente. Un reciente brote del virus H7N3 en el estado occidental de Jalisco, México, infectó a unos 3.4 millones de pollos y obligó a sacrificar y eliminar una cifra informada de 2.5 millones de aves, lo cual redujo el consumo de pollos y huevos y provocó un alza de precios (AFP, 2012).

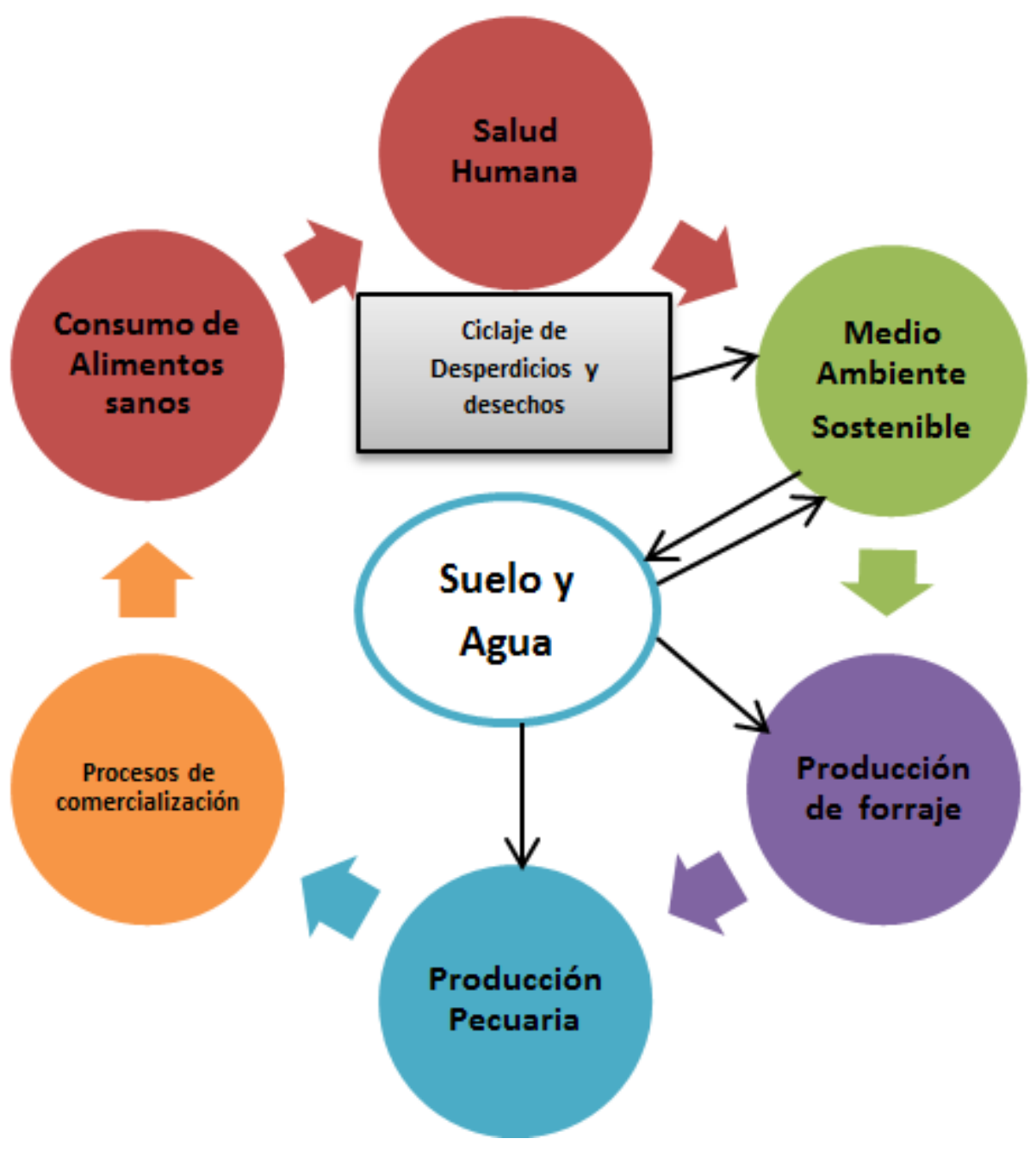

Figura 3. Relación entre salud y cadena alimentaria 


\section{Recursos naturales y clima}

A medida que se aumenta la producción ganadera también lo hacen los desafíos ambientales, especialmente en lo referente a tasas de deforestación (Wassenaar et al., 2007), para abordar la extensa degradación del suelo en el pasado y la potencial a futuro, Steinfeld et al., (2006) recomiendan programas de conservación de suelos, prácticas de silvopastoreo y mejor uso de los sistemas de pastoreo, también afirman que una intensificación sostenible de la producción de cultivos y ganado, reduciría la deforestación y la degradación de las praderas y las resultantes emisiones de dióxido de carbono. Para mejorar la calidad del agua en la región, sugieren una mejor gestión de los desechos del ganado en sistemas intensivos.

La ganadería tiene un $30 \%$ del uso de la tierra, generando el $14.5 \%$ de emisiones efecto invernadero de las cuales el $80 \%$ provienen directamente de los rumiantes, además utiliza el 8 a 15\% del agua global, teniendo impacto en su calidad y sus ciclos, generando disminución de biodiversidad, en donde las áreas marginales son más susceptibles al cambio climático. Cabe destacar que cierta ganadería se adapta con más "naturalidad" aprovechando cambios en las biomasas, mediante la adopción de sistemas intensivos, generando alta variabilidad en la intensidad de emisiones dependiendo de los diferentes sistemas productivos y entre regiones. Se ha demostrado que se puede mitigar 30\% de emisiones de dióxido de carbono realizando prácticas más eficientes y mejor provecho de los recursos (CEPAL et al., 2013).

\section{CINCO PRINCIPIOS PARA UNA GANADERÍA SOSTENIBLE}

\section{Incrementar la eficiencia}

Se debe mejorar la eficiencia de los recursos en su uso, tales como tierras, suelos, agua y nutrientes, disminuyendo la intensidad en la emisiones de $\mathrm{CO}_{2}$ por unidad de producto, reduciendo desperdicios a través del ciclaje y recuperación de nutrientes y energía, al mismo tiempo que se aprovechan desperdicios, estiércol, 
aguas negras $\mathrm{y} / \mathrm{o}$ riegos, $\mathrm{y}$ finalmente disminuyendo la sobrecarga de la cronicidad de las enfermedades endémicas y amenazas emergentes.

\section{Realzar los medios de vida y bienestar}

Es indispensable proteger los ahorros y bienes, aprovechando las funciones múltiples de la ganadería, para ello se debe desarrollar cadenas de valores, participación y competitividad de los actores. Además es necesario reconocer los valores culturales tales como culinaria, preferencias, costumbres y tradiciones, paralelamente garantizando el acceso a medicinas y vacunas de calidad.

\section{Proteger los recursos naturales}

No se debe dejar de lado el principio de producir más a partir de menos recursos en una forma que beneficie a todos, reduciendo la contención entre el alimento y la producción de piensos y forrajes. Es fundamental limitar la expansión de la ganadería en ecosistemas con gran valor, para ello es indispensable la gestión de tierras en forma integrada (planes de ordenamiento y destinación territorial), realzando y protegiendo recursos de agua y riegos, además identificar y maximizar recursos genéticos, protegiendo el bienestar animal. Además reducir los riesgos de enfermedades, en particular las amenazas de pandemia, para lo cual es necesario mejorar la capacidad de hacer frente a través de intervenciones técnicas e institucionales.

\section{Mejorar la gobernanza}

Para tener una ganadería sostenible es fundamental mejora el uso de bienes globales comunes (clima), y locales (pastoreo y agua), así como la participación en la gestión de recursos y las cadenas de valor, fortaleciendo la regulación, incentivos y marcos de inversión, garantizando sistemas y servicios veterinarios, integrados con los sectores de salud humana y salud del medio ambiente (una sola salud). 


\section{Inversiones actuales}

La expansión del sector ganadero debe estar acorde con la demanda de mercado, mientras que la regulación deficiente que es generada por los actuales vacíos institucionales tiene que eliminarse, además parte de los recursos deben destinarse a mejorar en la productividad (genética, alimentación y procesamiento). Así mismo, otra parte debe destinarse a crear oportunidades para los pequeños productores y ambientes silvopastoriles, buenas prácticas para mitigar los efectos del cambio climático, garantizar la bioseguridad y dietas saludables.

\section{RECOMENDACIONES PARA AGRICULTURA FAMILIAR Y LA GANADERÍA SOSTENIBLE PARA PEQUEÑOS PRODUCTORES}

Se debe caracterizar la agricultura familiar diseñando políticas diferenciadas, induciendo sistemas silvopastoriles, promoviendo instancias focalizadas en formalizar el sector (información, registro), desarrollando estrategias multisectoriales e intergubernamentales, fortaleciendo la asociatividad, alianzas y esquemas inclusivos: clave para inserción y finalmente adaptar la agricultura familiar y la ganadería sostenible al cambio climático, pero todo esto no será posible si no se forma y retiene una generación de relevo, priorizando jóvenes profesionales del sector agropecuario.

\section{CONCLUSIÓN}

La discrepancia entre el desarrollo de la ganadería y su impacto ambiental requiere un enfoque decidido pero equilibrado a la vez, considerando inversiones en investigaciones, infraestructura, prevención y erradicación de enfermedades, educación y capacitación, y una serie de medidas para mejorar la productividad y la rentabilidad de esta actividad.

\section{REFERENCIAS BIBLIOGRÁFICAS}

1. AFP (Agence France-Presse). Mexico kills $2.5 \mathrm{mn}$ poultry to contain bird flu. 2012. Recuperado 11 Julio 2014. Disponible En: http://www.google.com/hostednews/afp/article/ALeqM5hOw9R4VYbA zOTbfr3 uKSNNrmxUw?docld=CNG.e3bb940ba7cd96953abc2a7998969c09.261 
2. Blayney D, Gehlhar M, Bolling $\mathrm{CH}$, Jones K, Langley S, Normile MA, Somwaru A. U.S. Dairy at a Global Crossroads. Economic Research Report No. ERR-28, U.S. Department of Agriculture. Washington, D.C. 2006. Disponible En: http://webarchives.cdlib.org/sw15d8pg7m/http://ers.usda.gov/Publications/err2 $\underline{8 /}$

3. CEPAL (Comisión Económica Para América Latina y el Caribe), FAO (Organización de las Naciones Unidas para la Alimentación y la Agricultura), IICA (Instituto Interamericano de Cooperación para la Agricultura). Perspectivas de la agricultura y del desarrollo rural en las Américas: Una mirada hacia América Latina y el Caribe. 176 p. 2012.

4. FAO (Organización de las Naciones Unidas para la Alimentación y la Agricultura. FAOSTAT. Roma, IT. 2013. Disponible En: http://faostat.fao.org/site/291/default.aspx

5. FEDESARROLLO (Fundación para educación superior y el desarrollo), SAC (Sociedad de Agricultores de Colombia), INCODER (Instituto Colombiano de Desarrollo Rural), FINAGRO (Fondo para el financiamiento del sector agropecuario) y Banco Agrario. Políticas para el desarrollo de la agricultura en Colombia. Sociedad de Agricultores de Colombia. La Imprenta Editores S.A. 234 p. 2013.

6. FEDEGAN (Fondo Nacional de Ganaderos). Análisis del inventario Ganadero Colombiano, Comportamiento y variables explicativas. 21 p. 2013. Recuperado 12 diciembre $2014 . \quad$ Disponible En: http://www.fedegan.org.co/publicacion-presentaciones/analisis-del-inventarioganadero-colombiano-comportamiento-y-variables

7. Gómez JD, Rueda RA. Productividad del sector ganadero bovino en Colombia durante los años 2000 a 2009. Trabajo de grado, Colegio Mayor Nuestra Señora del Rosario, 89 p. 2011.

8. IICA (Instituto Interamericano de Cooperación para la Agricultura). Traceability, a source of pride for Uruguay's livestock subsector. Sowing innovation to harvest prosperity. San José, Costa Rica. 2013. Disponible En: http://www.iica.int/Eng/prensa/pages/ComunicadoPrensav1.aspx?cp=823

9. Martins L. Pig health issues: Overview of Latin America. 2011. PigProgress,net. Recuperado 31 Marzo 2014. Disponible En: http://www.pigprogress.net/background/pig-health-issues-overview-of-latinamerica-7801.html

10. Nash J. The greening (?) of agriculture in Latin America. Latin American \& the Caribbean: Opportunities for all. World Bank. 2012. Recuperado 9 Julio 2014. Disponible En: http://blogs.worldbank.org/latinamerica/node/633

11. ODEPA (Oficina de Estudios y Políticas Agrarias). Agricultura chilena 2014, Una perspectiva de mediano plazo. Ministerio de Agricultura, Gobierno de Chile. Santiago de Chile, Ed MAVAL Ltda, 242 p. 2005.

12. OCDE (Organización para la Cooperación y Desarrollo Económico) y FAO (Organización de las Naciones Unidas para la Agricultura y la Alimentación). 2014. Mercado de ganados y carnes, Proyecciones 2023. p. 31.

13. OCDE (Organización para la Cooperación y Desarrollo Económico) y FAO (Organización de las Naciones Unidas para la Agricultura y la Alimentación). Perspectivas Agrícolas 2011-2020, OECD Publishing y FAO. 225 p. 2011. 
14. OCDE (Organización para la Cooperación y el Desarrollo Económico) y FAO (Organización de las Naciones Unidas para la Agricultura y la Alimentación). Agricultural Outlook 2012-2021. OECD Publishing and FAO. 2012. Disponible En: http://dx.doi.org/10.1787/agr outlook-2012-en

15. Otte J, Costales A, Upton M. Smallholder livstock keepers in the era of globalization. Pro-Poor Livestock Policy Initiative, Living from Livestock Research Report RR Nr.05-06. UK, University of Reading, Earley Gate, Reading. 2005.2 Disponible En: http://www.fao.org/ag/againfo/programmes/en/pplpi/docarc/rep0506 globalisation livestock.pdf

16. Perry BD, Grace D, Sones K. Current drivers and future directions of global livestock disease dynamics. Proceedings of the National Academy of Sciences of the United States of America. 2011. Disponible En: http://www.pnas.org/content/early/2011/05/10/1012953108.abstract

17. Pica G, Pica U, Otte, J. The livestock sector in the World Development Report 2008: Re-assessing the policy priorities. Living from livestock research report no. 08-07. Pro-Poor Livestock Policy Initiative, Food and Agriculture Organization, Roma, IT. 2008. Disponible En: http://www.fao.org/ag/againfo/programmes/en/pplpi/docarc/rep 0807 WDRLiv estock UP et al 080805.pdf.16161612

18. Steinfeld, H., Gerber, P., Wassenaar, T., Castel, V., Rosales, M., de Haan, C. Livestock's Long Shadow: Environmental Issues and Options. Rome, FAO. 2006.

19. Thornton PK, Kruska RL, Henninger N, Kristjanson PM, Reid RS, Atieno F, Odero AN, Ndegwa T. Mapping poverty and livestock in the developing world. ILRI (International Livestock Research Institute), Nairobi, Kenya. 2002. Disponible http://www.ilri.org/InfoServ/Webpub/fulldocs/InvestAnim/Book2/media/PDF ch apters/B2 Front.pdf

20. UPI (United Press International). South America cattle outbreak threat lingers. 2012. Disponible En: http://www.upi.com/Business News/EnergyResources/2012/06/28/S-America-cattle-outbreak-threat-lingers/UPI$26911340900875 /$

21. Wassenaar T, Gerber P, Verburg PH, Rosales M, Ibrahim M, Steinfeld H. Projecting land use changes in the Neotropics: the geography of pasture expansion into forest. Global Environmental Change, 17: 86-104. 2007. 